\title{
Primary diffuse large B-cell lymphoma of the dura with skull and scalp involvement: A case report and brief review of the literature
}

\author{
ZHONG-WEN LV ${ }^{1 *}$, KAI-LIANG CHENG ${ }^{1 *}$, HONG-JI TIAN $^{2}$ and XUE-MEI HAN ${ }^{2}$ \\ Departments of ${ }^{1}$ Radiology and ${ }^{2}$ Neurology, China-Japan Union Hospital of Jilin University, \\ Changchun, Jilin 130031, P.R. China
}

Received January 18, 2015; Accepted February 24, 2016

DOI: $10.3892 / \mathrm{ol} .2016 .4455$

\begin{abstract}
Primary dural lymphoma(PDL) refers to a lymphoma with epidural or subdural involvement and is a rare subtype of primary central nervous system lymphoma. Diffuse large B-cell lymphoma (DLBCL) presenting as PDL is extremely rare. The present study reports a case of PDL with skull and scalp involvement in a 56-year-old man. Magnetic resonance imaging (MRI) revealed that the tumor was located under the right parietal inner plate and was attached to the dura mater. Following contrast-enhanced MRI, markedly enhanced tumor signals were observed, and mild homogeneous enhancement was observed in the diploë and soft tissues under the scalp, near the parietal bone. Under general anesthesia, the patient underwent craniotomy and tumor resection. The postoperative pathological diagnosis was DLBCL. Tumors were additionally identified inside the skull and subcutaneous tissues. The patient was administered chemotherapy postoperatively, and the prognosis subsequent to the 4-year follow-up was favorable. Primary malignant lymphoma should be considered in the differential diagnosis of scalp masses and meningeal lesions. Early diagnosis and individualized treatment is closely associated with a favorable outcome.
\end{abstract}

\section{Introduction}

Primary central nervous system lymphoma (PCNL) is a malignant type of non-Hodgkin's lymphoma that is only observed in the central nervous system (1). It is a rare extranodal lymphoma accounting for 1-6\% of all intracranial tumors, and 1-2\% of all malignant lymphomas worldwide (2). PCNL has a relatively high incidence rate among patients with acquired immune

Correspondence to: Dr Xue-Mei Han, Department of Neurology, China-Japan Union Hospital of Jilin University, 2 Xian Tai Avenue, Changchun, Jilin 130031, P.R. China

E-mail: xuemeihan1971@163.com

*Contributed equally

Key words: magnetic resonance imaging, dura lymphoma, diffuse large B-cell lymphoma, chemotherapy deficiency syndrome, organ transplant recipients and patients with immunodeficiency (3).

Primary dural lymphoma (PDL) refers to a rare subtype of PCNL with epidural or subdural involvement that is diagnosed based on imaging (4). It accounts for $<1 \%$ of all brain lymphomas and $\sim 0.1 \%$ of all non-Hodgkin's lymphomas globally (5). PDL originates outside the central nervous system of immunologically stable individuals.

Diffuse large B-cell lymphoma (DLBCL) is a subtype of PCNL that is pathologically diagnosed (4). The presentation of DLBCL as PDL is extremely rare, and may result in a misdiagnosis of meningioma or other dural-based neoplasm (4). The neuroimaging findings of DLBCL of the dura and PDL are similar to invasive meningioma; DLBCL of the dura and PDL present with extra-axial lesions that appear iso- or hypointense on T1-weighted magnetic resonance imaging (MRI) and diffusely enhance with gadolinium administration $(6,7)$. Furthermore, a dural tail is a frequent finding in meningiomas and PDL (8). However, underlying vasogenic edema appears to be more common in PDL. Thus, the differential diagnosis of PDL based on neuro-imaging is important.

PDL or DLBCL presenting as PDL is a very rare disease and there is no standard treatment. As extranodal diseases limited to a single site, they respond favorably to surgery or focal radiation. Adjuvant treatment is necessary in the majority of cases (6). Various treatment combinations, including complete surgical resection, systemic chemotherapy (CHOP and rituximab) and adjuvant radiation therapy have been attempted in cases of primary DLBCL (9).

PDL is more indolent and has a better prognosis than parenchymal primary CNS lymphoma or systemic lymphoma with CNS metastasis (10). In general, patients with PDL have a favorable outcome, with a 5-year overall survival rate of $>86 \%$. The DLBCL relapse rate among all patients with complete remission status ranges between 7.0 and $20.0 \%$, with a weighted summary proportion of $13.7 \%$. The overall survival (OS) of patients with DLBCL is $83 \%$. In addition, the recurrence rates of invasive meningioma are high (11).

The present study reports a case of DLBCL of the dura mater with skull and scalp involvement, presenting as PDL, and includes a brief review of the literature. The diagnosis made based on preoperative imaging was malignant meningioma. Following surgery, the patient received systematic chemotherapy for 4 cycles, and the prognosis subsequent to 

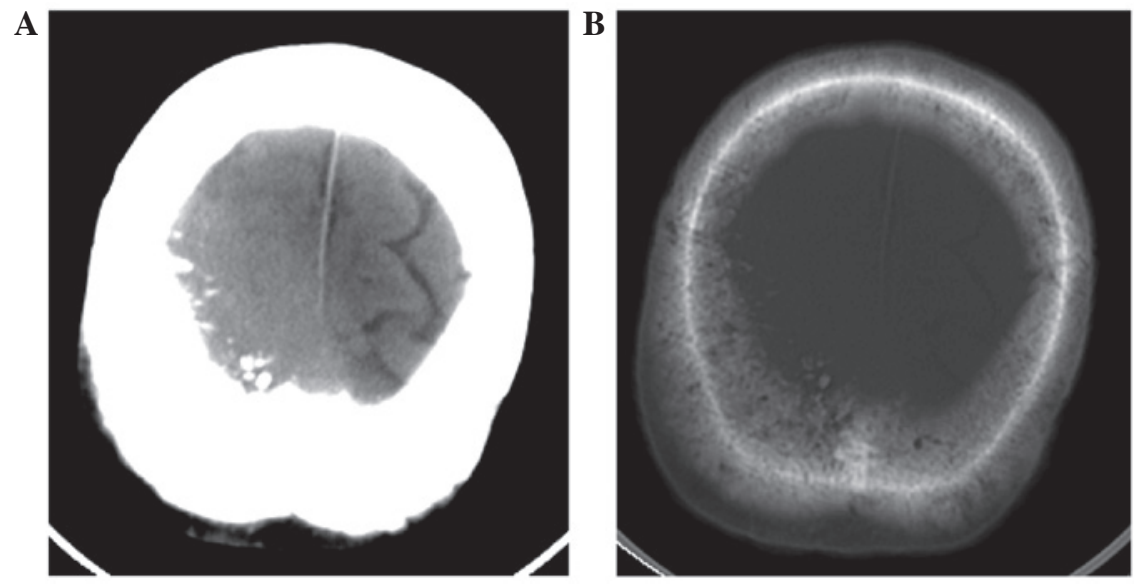

Figure 1. (A) Non-enhanced head computed tomography scan revealing a slightly high-density mass under the right parietal skull plate. (B) Bone window view showing rough inner plate of the right parietal bone.
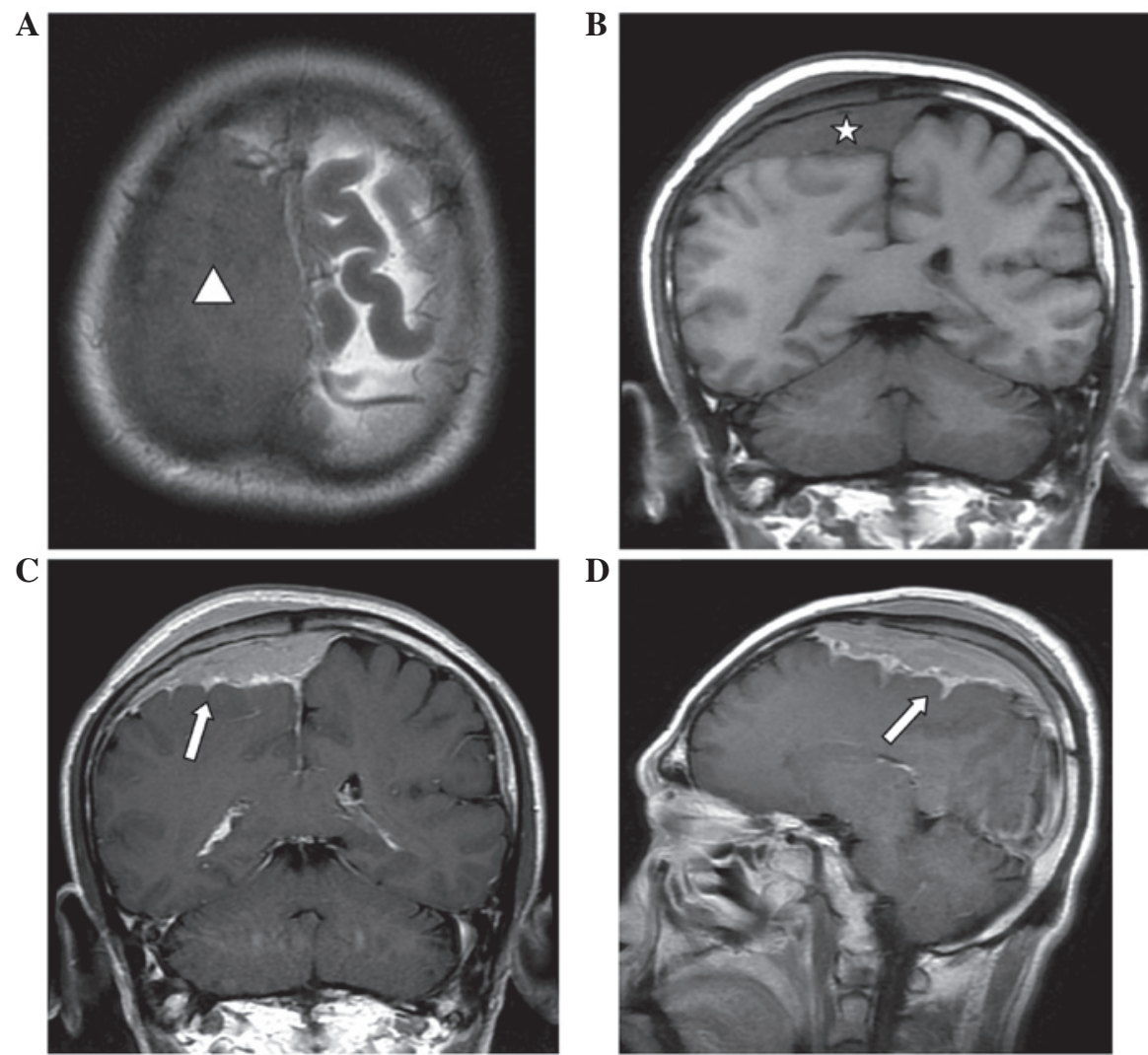

Figure 2. (A and B) Non-enhanced MRI scan revealing a right parietal dural mass (arrowhead and asterisk), with equal-low signal on T1WI and equal signal on T2WI compared with normal brain imaging. The signal near the parietal diploë was reduced, and subcutaneous soft tissues were observed to be mildly swollen. (C and D) Via enhanced MRI scanning the mass was observed to be markedly enhanced, demonstrating a relatively long dural tail sign at the edge. The pia mater was involved, exhibiting enhancements of the jagged shape (arrows), indicating leptomeningeal involvement, and moderate enhancement was observed in the adjacent parietal diploë and subcutaneous soft tissues. MRI, magnetic resonance imaging; WI, weighted image.

the 4-year follow-up was favorable. The study was approved by the ethics committee of the China-Japan Union Hospital of Jilin University (Changchun, China) and written informed consent was obtained from the patient.

\section{Case report}

Clinical information. A 56-year-old man presented to the China-Japan Union Hospital of Jilin University in
November 2010 with a 2-week history of intermittent headaches, dizziness and tinnitus with no clear cause. The headaches were described as dull and primarily confined to the parietal-occipital area, and resolved on their own. Upon physical examination, the patient was conscious and verbally fluent. His pupils were equal in size, round and reactive to light. Muscle strength and tone were normal, and no pathological reflexes were elicited. Superficial systemic lymph nodes were not found upon palpitation, and no abnormalities were identified 

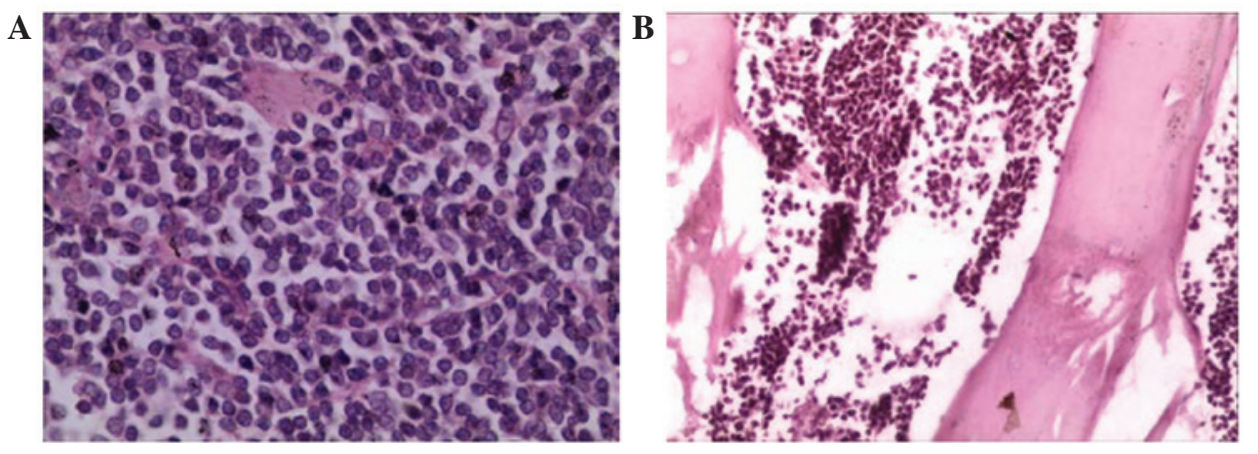

Figure 3. HE staining showing (A) diffuse large cell proliferation. (magnification, x40) and (B) skull involvement (magnification, x20). HE, hematoxylin and eosin.

A

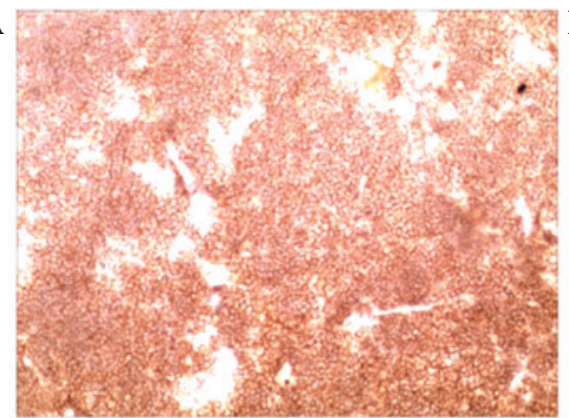

D

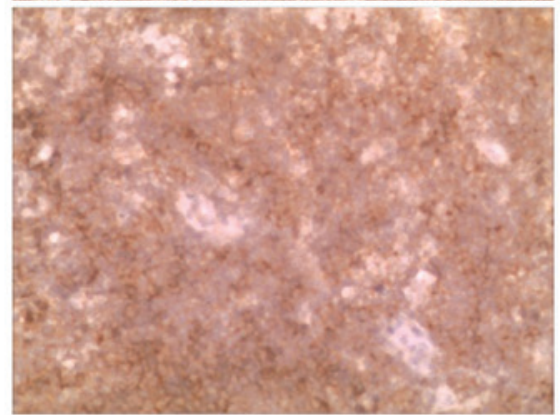

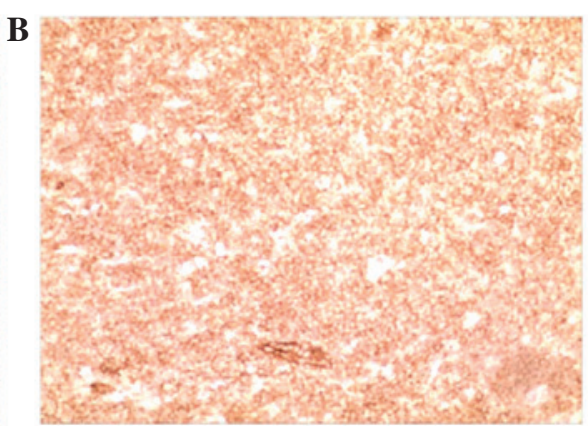

$\mathbf{E}$

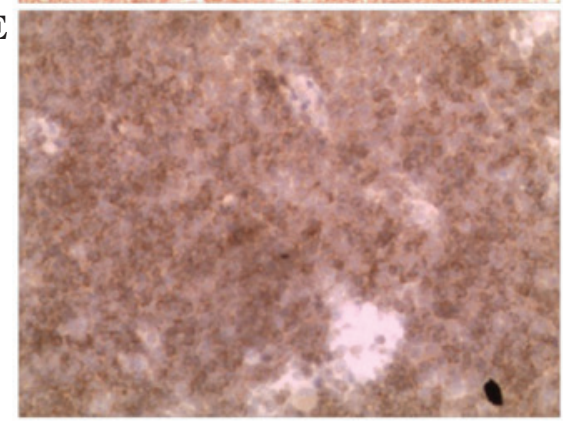

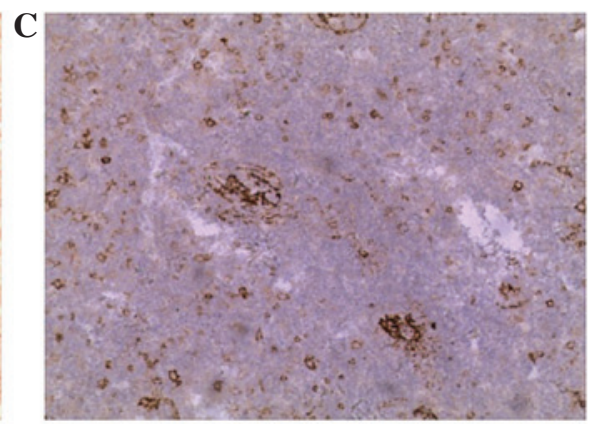

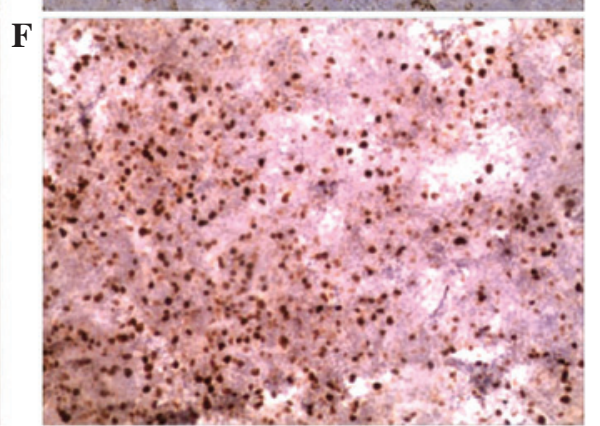

Figure 4. Immunohistochemistry results. (A) CD20 diffusely positive expression (magnification, x10), (B) CD21 diffusely positive expression (magnification, x10), (C) CD23 positive expression (magnification, x10), (D) CD79 positive expression (magnification, x20), (E) B-cell lymphoma 2 positive expression (magnification, x20), (F) Ki-67 positive expression (magnification, x10). Diffusely positive expression is indicated by $>75 \%$ positive cells; positive expression is indicated by $25-75 \%$ positive cells. $\mathrm{CD}$, cluster of differentiation.
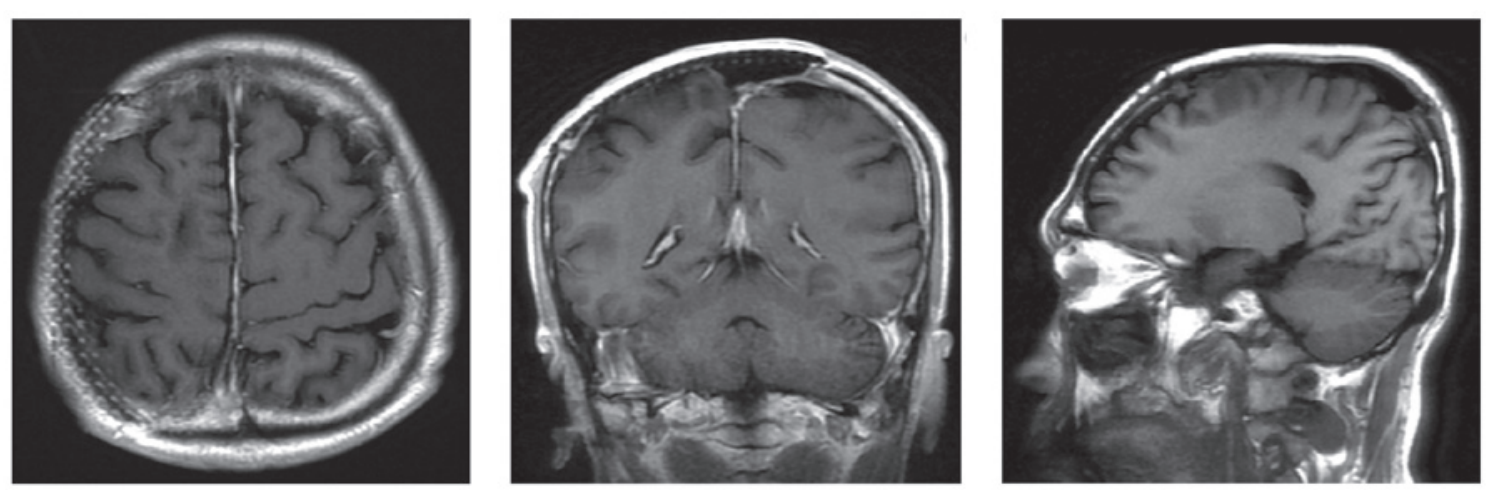

Figure 5. Postoperative examination enhanced magnetic resonance imaging scans revealing the implanted artificial cranial plate and no signs of tumor recurrence.

in the heart and lungs. Ultrasound examination additionally revealed no abnormalities in the superficial systemic lymph nodes. Routine blood [red blood cell count, 462 ×10\%/1 (normal range, 400-550x10\%/1); hemoglobin concentration,
$160 \mathrm{~g} / 1$ (normal range, 120-160 g/l); white blood cell count, $6.5 \times 10^{9} / 1$ (normal range, $4-10 \times 10^{9} / 1$ ); neutrophils, $65 \%$ (normal range, 50-70\%); lymphocytes, $35 \%$ (normal range, 20-40\%); platelet count, $180 \times 10^{9} / 1$ (normal range, 100-300x10 $/ 1$ )], urine 
(white blood cells, negative; blood, negative; specific gravity, 1.015 (normal range, 1.015-1.025); urobilinogen, $5 \mu \mathrm{mol} / 1$ (normal, <16 $\mu \mathrm{mol} / 1$ ); protein, negative; glucose, negative; ketones, negative], blood lactate dehydrogenase (110 IU/1; normal range, 80-245 IU/l) and $\beta 2$ microglobulin $(1,182 \mu \mathrm{g} / \mathrm{l}$; normal range, 604-2,282 $\mu \mathrm{g} / \mathrm{l})$ test results were normal.

Computed tomography (CT) scanning (Lightspeed Pro16; GE Healthcare, Chalfont, UK) of the head revealed a slightly high-density mass beneath the right parietal skull plate, without clear boundaries (Fig. 1A). The tumor presented as a shadow with relatively uniform density that grew across the cerebral falx. The bone window view revealed a rough inner plate of the right parietal bone, and scattered high-density patches (Fig. 1B). MRI (Signa HDx 1.5T; GE Healthcare) revealed that a wide-base tumor attached to the dura mater was located under the right parietal inner plate, and was growing across the dural midline to the left (Fig. 2). Mild swelling of the soft tissues was observed near the scalp, and the signals within the tumor were relatively uniform, with equal-low signal on T1-weighted imaging (WI), equal signals on T2WI and fluid-attenuated inversion recovery and reduced tumor signals near the parietal diploë compared with normal brain imaging. Via contrast-enhanced MRI, tumor signals were observed to be markedly enhanced, demonstrating a relatively long dural tail sign at the edge. The lesion was observed to invade into the pia mater, with enhancements of a jagged shape in the right parietal sulci, indicating leptomeningeal involvement, as well as mild homogeneous enhancement in the diploë and soft tissues under the scalp, near the parietal bone. The diagnosis made based on preoperative imaging was invasive meningioma.

Surgery and pathology. Under general anesthesia, the patient underwent craniotomy and tumor resection. During the surgery, it was observed that the tumor was located in the dura mater, and was pale gray, firm and adjacent to the pia mater, with skull and skin involvement. Resected tissues were fixed in 10\% formalin (Jinan Baibo Biotechnology Co., Ltd., Jinan, China) and paraffin (Shanghai Hualing Recovery Equipment Factory, Shanghai, China)-embedded, cut into 1-3 $\mu \mathrm{m}$ sections, stained with hematoxylin and eosin [Huayueyang Biotechnology (Beijing) Co., Ltd., Beijing, China] and observed under a microscope (CX31; Olympus Corporation, Tokyo, Japan). The postoperative pathological diagnosis was DLBCL. Tumors were additionally observed inside the skull and subcutaneous tissues, as revealed by hematoxylin and eosin staining (Fig. 3). These tumors were resected, and cranioplasty using titanium mesh was performed. Immunohistochemistry was performed mouse monoclonal antibodies against cluster of differentiation (CD)21 (cat no. EP3093), CD23 (cat no. SP23), CD79 (cat no. SP18), B-cell lymphoma-2 (cat. no. MAB-0014) and Ki-67 (cat no. MX006) (Fuzhou Maixin Biotech. Co., Ltd., Fuzhou, China), all at dilutions of 1:100. The results revealed elevated expression of CD21, CD20, B-cell lymphoma 2, CD23, CD79 and $<30 \% \mathrm{Ki}-67$, indicating a low cell proliferation index (Fig. 4).

Postoperative follow-up. Following surgery, the patient underwent adjuvant cyclophosphamide, hydroxydaunorubicin, Oncovin $^{\circledR}$, prednisone (CHOP) chemotherapy every 3 weeks for 4 cycles $\left(750 \mathrm{mg} / \mathrm{m}^{2}\right.$ cyclophosphamide intravenously on day $1 ; 50 \mathrm{mg} / \mathrm{m}^{2}$ adriamycin intravenously on day $1 ; 1.4 \mathrm{mg} / \mathrm{m}^{2}$ vincristine intravenously on day 1 ; and $60 \mathrm{mg}$ prednisone orally on days 1-5). The patient remained in a good condition 26 months subsequent to the surgery, with no neurological abnormalities. Subsequently the patient underwent artificial cranial plate implantation. A total of 50 months subsequent to resection of the tumor, no signs of tumor recurrence were detected by MRI (Fig. 5).

\section{Discussion}

PCNL has a relatively high incidence among patients with acquired immune deficiency syndrome, organ transplant recipients and patients with immunodeficiency (3). PDL is a rare type of PCNL and accounts for $0.6 \%$ of all intracranial tumors (12). PDL originates in the dura mater, and differs from other central nervous system lymphomas in its biological mechanism (13). PDL is typically a low-level marginal zone BCL, whereas other types of central nervous system lymphomas are high-level diffuse large BCLs (14). The majority of patients with PDL do not exhibit immune system abnormalities, unlike patients with primary malignant lymphoma of the brain, who are known to experience immune dysfunction (8). Patients with PDL frequently present with headaches, scalp swelling, meningeal irritations, epilepsy and symptoms of cranial nerve involvement (15).

Murray et al (16) conducted a retrospective analysis of 693 cases with brain lymphoma, and reported that the mean age at diagnosis was 52 years (range, 30-65 years), with a male to female ratio of $1.5: 1$, values similar to those for systemic lymphoma (17). By contrast, Shoko et al (18) examined 25 cases of dural malignant BCL, and reported the mean age to be 48.7 years (range, 28-66 years), a value that is similar to that of primary brain lymphoma; however, the male to female ratio was 2:23, markedly different from that of systemic lymphomas. The patient in the present study was diagnosed with primary DLBCL, based on the findings of histology and immunohistochemistry. Pathological examination revealed scalp and skull involvement, which is rare. Due to the lack of lymphatic tissue in the dura, the pathogenesis of PDL remains unclear, but several hypotheses have been proposed, including chronic inflammation, chronic infection, autoimmune diseases and meningeal epithelial components $(8,19,20)$.

As PDL lacks typical tumor imaging manifestations, its diagnosis is considerably challenging (21). Non-enhanced CT scans typically reveal a mass of equal or slightly higher density compared with normal brain imaging and, in cases of skull involvement, CT scans with the bone window setting show a rough cranial plate or clear bone damage (21). Non-enhanced MRI scans typically reveal equal or slightly higher T1WI signals, and diffusion-weighted imaging frequently shows a relatively high signal (21). In cases of brain parenchyma involvement, varying degrees of edema may be observed upon T1- and T2-weighted imaging (8); skull involvement is manifested by a reduced signal in the diploë. In the present study, enhanced MRI revealed the tumor to be markedly enhanced, exhibiting a relatively long dural tail sign in the periphery. The diffusion may be restricted, reflecting compact cellularity (21). Leptomeningeal involvement may be identified by enhancement 
of a jagged shape in adjacent sulci upon MRI. PDL tends to be located in areas that are rich in meningeal cells (22), and frequently forms a focal lump or plaque-like thickening of the dura mater, thereby producing imaging manifestations similar to those of other meningeal lesions (23). The differential diagnosis of PDL often includes epidural hematoma, anaplastic meningioma, hemangiopericytoma, meningeal metastasis and meningeal sarcoma $(8,24)$. Menniti et al $(25)$ retrospectively reviewed 14 cases of PDL previously reported in the literature. Out of these 14 cases, a single case was initially diagnosed as a subdural hematoma, while the remaining cases were diagnosed as meningiomas. The body of PDL is typically relatively flat, with a fairly long dural tail sign (24). Intratumoral calcification is rarely observed, and hyperplasia or hardening of the adjacent skull bone is almost never exhibited; thus, PDL can be differentiated from meningiomas (21). A history of trauma may assist with the differentiation between epidural hematoma and PDL; however, Iaccarino et al (26) reported a rare clinical case of PDL, with clinical symptoms and imaging results similar to those of chronic epidural hematoma. They additionally reviewed 4 cases of PDL that were misdiagnosed as chronic subdural hematomas, in 2 cases of which chronic subdural hematoma and PDL coexisted, making the diagnosis challenging (26). When imaging techniques are unable to provide a clear diagnosis, pathological examination, following craniotomy or directed biopsy, is the only approach to confirm the diagnosis (27).

Due to the paucity of cases in individual series, no standard treatment has been established for intracranial primary DLBCL. Various treatment combinations, including complete surgical resection, systemic chemotherapy (CHOP and rituximab) and adjuvant radiation therapy have been attempted in cases of primary DLBCL $(6,9,28,29)$. Despite the success of surgical resection of the tumor in the present case, postoperative adjuvant chemotherapy was also employed. Radiotherapy was not offered as part of the treatment to avoid neurotoxicity, considering that this patient may potentially achieve long-term survival. The reasons for the selection of systemic chemotherapy as a treatment option were the involvement of the skull and scalp, and the fact that the chemotherapy drugs had free access to the PDL and did not need to pass through the blood-brain barrier. There is no definitive answer to whether radiotherapy should have been administered to the present patient. It was considered that the neurotoxicity caused by radiotherapy was too great a risk for a healthy patient, and should only be used as salvage treatment in case of relapse (30).

The prognosis of malignant B-cell-type dural lymphoma is relatively good. Surgical excision is considered adequate for the treatment of the disease, regardless of whether postoperative local radiotherapy is performed (31). Shoko et al (18) reviewed 21 cases of malignant B-cell-type dural lymphoma, and observed that the average survival time of patients, including 19 patients who remained alive when the paper was published, was 29.3 months. By contrast, the survival time of non-Hodgkin's central nervous system tumor patients was observed to be $12-18$ months (17), with only $8 \%$ of patients surviving for $>3$ years (32). Thus, the favorable outcome of the present case is similar to that of previous case reports.

In conclusion, the present study described a rare case of DLBCL presenting as PDL with skull and scalp involvement, which was diagnosed based on preoperative imaging and pathology. Follow-up non-enhanced CT scans, as well as non-enhanced and enhanced MRI scans, were required. Early diagnosis and treatment is crucial for patients with DLBCL presenting as PDL; therefore, upon the detection of a diffuse infiltrative dural lesion, additional examinations should be performed to confirm or rule out PDL. Despite its rarity, primary malignant lymphoma should be considered in the differential diagnosis of scalp masses. Treatment should be individualized and should involve a multidisciplinary team approach, including neurosurgery, radiation and oncology professionals.

\section{Acknowledgements}

The present study was financially supported by the International Cooperation Projects of the Science and Technology Agency of Jilin Province (grant no. 20130413027GH) and the Natural Science Foundation of Jilin Province (grant no. 20160101023JC)

\section{References}

1. Ervin T and Canellos GP: Successful treatment of recurrent primary central nervous system lymphoma with high-dose methotrexate. Cancer 45: 1556-1557, 1980.

2. Abrey LE, Yahalom J and DeAngelis LM: Treatment for primary CNS lymphoma: The next step. J Clin Oncol 18: 3144-3150, 2000.

3. Ferreri AJ, Abrey LE, Blay JY, Borisch B, Hochman J, Neuwelt EA, Yahalom J, Zucca E, Cavalli F, Armitage J and Batchelor T: Summary statement on primary central nervous system lymphomas from the Eighth international conference on Malignant Lymphoma, Lugano, Switzerland, June 12 to 15, 2002. J Clin Oncol 21: 2407-2414, 2003.

4. DeAngelis LM: Brain tumors. N Engl J Med 344: 114-123, 2001.

5. Plotkin S and Batchelor T: Primary nervous-system lymphoma. Lancet Oncol 2: 354-365, 2001.

6. Said R, Rizk S and Dai Q: Clinical challenges of primary diffuse large B-cell Lymphoma of the dura: Case report and literature review. ISRN Hematol 2011: 945212, 2011.

7. Brito ABC, Reis F, de Souza CA, Vassallo J and Lima CSP: Intracranial primary dural diffuse large B-cell lymphoma successfully treated with chemotherapy. Int J Clin Exp Med 7: 456-460, 2014.

8. Iwamoto FM and Abrey LE: Primary dural lymphomas: A review. Neurosurg Focus 21: E5, 2006.

9. Reis F, Schwingel R, Queiroz Lde S and Zanardi Vde A: Primary dural lymphoma: A rare subtype of primary central nervous system lymphoma (PCNSL). Arq Neuropsiquiatr 69: 264-265, 2011.

10. Thieblemont C, Berger F, Dumontet C, Moullet I, Bouafia F, Felman P, Salles G and Coiffier B: Mucosa-associated lymphoidtissue lymphoma is a disseminated disease in one third of 158 patients analyzed. Blood 95: 802-806, 2000

11. Adams HJ, Nievelstein RA and Kwee TC: Prognostic value of complete remission status at end-of-treatment FDG-PET in R-CHOP-treated diffuse large B-cell lymphoma: Systematic review and meta-analysis. Br J Haematol 170: 185-191, 2015.

12. Yamada SM, Ikawa N, Toyonaga S, Nakabayashi H, Chang Park K and Shimizu K: Primary malignant B-cell-type dural lymphoma: Case report. Surg Neurol 66: 539-543, 2006.

13. Benouaich A, Delord JP, Danjou M, Richaud J, Urocoste E, Soum F, Aziza R and Roche H: Primary dural lymphoma: A report of two cases with review of the literature. Rev Neurol (Paris) 159: 652-658, 2003 (In French).

14. Venkataraman G, Rizzo KA, Chavez JJ , Streubel B, Raffeld M, Jaffe ES and Pittaluga S: Marginal zone lymphomas involving meningeal dura: Possible link to IgG4-related disease. Mod Pathol 24: 355-366, 2011.

15. Galarza M, Gazzeri R, Elfeky HA and Johnson RR II: Primary diffuse large B-cell lymphoma of the dura mater and cranial vault. Case report and literature review. Neurosurg Focus 21: E10, 2006. 
16. Murray K, Kun L and Cox J: Primary malignant lymphoma of the central nervous system. Results of treatment of 11 cases and review of the literature. J Neurosurg 65: 600-607, 1986.

17. Parekh HC, Sharma RR, Keogh AJ and Prabhu SS: Primary malignant non-Hodgkin's lymphoma of cranial vault: A case report. Surg Neurol 39: 286-289, 1993.

18. Shoko M, Ikawa N, Toyonaga S, Nakabayashi H, Chang Park K and Shimizu K: Primary malignant B-cell-type dural lymphoma: Case report. Surg Neurol 66: 539-543; Discussion 543, 2006.

19. Kumar S, Kumar D, Kaldjian EP, Bauserman S, Raffeld M and Jaffe ES: Primary low-grade B-cell lymphoma of the dura: A mucosa associated lymphoid tissue-type lymphoma. Am J Surg Pathol 21: 81-87, 1997.

20. Isaacson PG and Du MQ: MALT lymphoma: From morphology to molecules. Nat Rev Cancer 4: 644-653, 2004.

21. Jacobs A, Kracht LW, Grossman A, Ruger MA,Thomas AV, Thiel A and Herholz K: Imaging in neurooncology. NeuroRx 2: 333-347, 2005.

22. Goetz P, Lafuente J, Revesz T, Galloway M, Dogan A and Kitchen N: Primary low-grade B-cell lymphoma of mucosa-associated lymphoid tissue of the dura mimicking the presentation of an acute subdural hematoma. Case report and review of the literature. J Neurosurg 96: 611-614, 2002.

23. Johnson MD, Powell SZ, Boyer PJ, Weil RJ and Moots PL: Dural lesions mimicking meningiomas. Hum Pathol 33: 1211-1226, 2002

24. Sotoudeh $\mathrm{H}$ and Yazdi HR: A review on dural tail sign. World J Radiol 2: 188-192, 2010.

25. Menniti A, Moschettoni L, Liccardo G and Lunardi P: Low-grade primary meningeal lymphoma: Case report and review of the literature. Neurosurg Rev 28: 229-233, 2005.
26. Iaccarino C, Schiavi P, Crafa P, Bronzoni C, Ramponi V, Mantenuto G, Cavanna L and Servadei F: Primary dural lymphoma mimicking a chronic epidural hematoma. Differential diagnosis of two rare conditions. Clin Neurol Neurosurg 115: 1510-1513, 2013.

27. Nishimoto T, Yuki K , Sasaki T , Imada Y, Murakami T and Kodama Y: A case of subcutaneous malignantlymphoma with dura mater lesion. No Shinkei Geka 31: 43-47, 2003 (In Japanese).

28. DeAngelis LM, Seiferheld W, Schold SC, Fisher B and Schultz CJ; Radiation Therapy Oncology Group Study: Combination chemotherapy and radiotherapy for primary central nervous system lymphoma: Radiation therapy oncology group study 93-10. J Clin Oncol 20: 4643-4648, 2002.

29. Giordano A, Perrone T, Guarini A, Ciappetta P, Rubini G, Ricco R, Palma M, Specchia G and Liso V: Primary intracranial dural B cell small lymphocytic lymphoma. Leuk Lymphoma 48: 1437-1443, 2007.

30. Sanjeevi A, Krishnan J, Bailey PR and Catlett J: Extranodal marginal zone B-cell lymphoma of malt type involving the cavernous sinus. Leuk Lymphoma 42: 1133-1137, 2001.

31. Horning SJ, Weller E, Kim K, Earle JD, O'Connell MJ, Habermann TM and Glick JH: Chemotherapy with or without radiotherapy in limited-stage diffuse aggressive non-Hodgkin's lymphoma: Eastern cooperative oncology group study 1484 J Clin Oncol 22: 3032-3038, 2004.

32. Freudenstein D, Bornemann A, Ernemann U, Boldt R and Duffner F: Intracranial malignant B-cell lymphoma of the dura. Clin Neuropathol 19: 34-37, 2000 